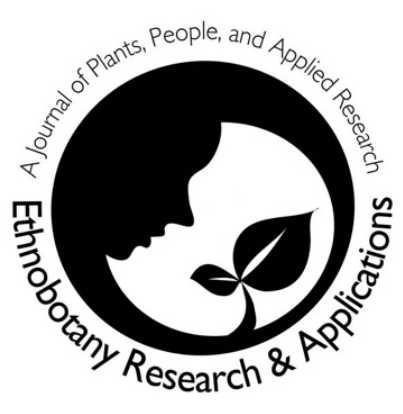

\title{
Synthèse et analyse de données sur les inventaires de plantes médicinales de Madagascar
}

Rafidison Verohanitra, Ratsimandresy Fabien,
Rakotondrajaona Roland, Rasamison Vincent,
Rakotoarisoa Marrino, Rakotondrafara Andriamalala,
Rakotonandrasana Stéphan Richard

\section{Databases and Inventories}

\begin{abstract}
Résumé
Contexte: Madagascar, une grande île de l'Océan indien est l'un des points chauds du monde en biodiversité. Parmi cette richesse biologique, les plantes médicinales tiennent une place importante dans la vie quotidienne des Malagasy. Cependant, la documentation sur les utilisations des plantes médicinales reste insuffisante et la synthèse des travaux effectués date de 1990.
\end{abstract}

Méthodes: L'étude se base sur la consultation de 99 publications. Les taxa les plus cités sont déterminés d'après les valeurs de leur fréquence de citation. Le niveau de fidélité des espèces pour chaque maladie est calculé selon la formule de Friedmann et ses collaborateurs.

Résultats: Les plantes médicinales recensées à Madagascar comptent 3245 espèces dont $60 \%$ sont endémiques. Croton L. (98 espèces), Helichrysum Mill. (46 espèces) Noronhia Stadtm. ex Thouars, (39 espèces) et Psychotria L. (29 espèces) sont les genres les mieux représentés. Albizia Durazz. (23,5\%), Aloe A. Rich. (22,7\%), et Aphloia (DC.) Benn. $(22,7 \%)$ sont les mieux cités.

Les trois premières familles les plus diversifiées coïncident avec celles des mieux citées, dont Fabaceae (279 espèces), Asteraceae (238 espèces), et Rubiaceae (218 espèces). Psiadia altissima (DC.) Drake $(36,4 \%)$ et Cinnamosma macrocarpa $\mathrm{H}$. Perrier $(32,3 \%)$ sont les deux premières espèces autochtones endémiques les plus citées, Harungana madagascariensis Lam. Ex Poir. (31,3\%) pour les espèces autochtones non endémiques et Lantana camara $L$. $(27,3)$ pour les introduites.
Les maladies de l'appareil digestif et de l'appareil génito-urinaire, les lésions traumatiques, les empoisonnements ainsi que certaines autres conséquences de causes externes constituent les premières maladies les plus soignées avec des plantes. Les niveaux de fidélité des 5 premières espèces utilisées pour traiter ces groupes de maladies se situent entre 50 à $95,5 \%$. Ces maladies appartiennent également à celles les plus morbides à Madagascar.

\section{Correspondence}

Rafidison Verohanitra', Ratsimandresy Fabien', Rakotondrajaona Roland ${ }^{2}$, Rasamison Vincent ${ }^{3}$, Rakotoarisoa Marrino², Rakotondrafara Andriamalala ${ }^{2}$, Rakotonandrasana Stéphan Richard $^{2^{*}}$

${ }^{1}$ Faculté des Sciences d'Antananarivo, Département de Biologie et Ecologie Végétales, BP 906, Antananarivo, Madagascar

${ }^{2}$ Centre National d'Application de Recherches Pharmaceutiques, BP 702, Ambodivoanjo, Rue RP Rahajarizafy, Antananarivo, Madagascar

3Institut d'Enseignement Supérieur Antsirabe Vakinankaratra.

*Corresponding author: Rakotonandrasana Stéphan Richard, stephanandrasana@yahoo.fr

\section{Ethnobotany Research \& Applications} 18:40 (2019)

Conclusion: Le présent travail permet de connaître l'état actuel des plantes médicinales de Madagascar et les maladies correspondantes. Ces données sont utiles pour influencer les futurs projets de conservation de la biodiversité à Madagascar, 
d'investigations pharmacologiques ethnobotaniques des plantes médicinales.

Mots clés: Madagascar, plantes médicinales, état actuel, espèces mieux représentées et mieux citées.

\begin{abstract}
Background: Madagascar, a big island in the Indian Ocean is one of the countries of hotspot biodiversity in the world. Among this biological richness, medicinal plants hold an important place in the everyday life of Malagasy people. However, the documentation on the uses of medicinal plants is still scarce and the synthesis of related works dated back to 1990 .
\end{abstract}

Methodes: This study was conducted by referring to 99 publications. The most cited taxa are determined according to the values of their frequency of citation. The fidelity level of species related to each disease is calculated using the formula of Friedmann et al.

Results: The medicinal plants inventoried in Madagascar consist of 3245 species, of which $60 \%$ are endemic. Croton L. (98 species), Helichrysum Mill. (46 species) Noronhia Stadtm. ex Thouars, (39 species) et Psychotria L. (29 species) are the most represented genera. Albizia Durazz. (23,5\%), Aloe A. Rich. (22,7 \%) and Aphloia (DC.) Benn. (22,7\%) are the most cited ones.

The first three most diversified families are the same as those the mostly cited, namely Fabaceae (279 species), Asteraceae (238 species), and Rubiaceae (218 species). Psiadia altissima (DC.) Drake and Cinnamosma macrocarpa $\mathrm{H}$. Perrier are the two first endemic autochthon species which are the most frequently cited. Harungana madagascariensis Lam. ex Poir. for the non endemic autochthon species and Lantana camara L. for the introduced species.

The digestive and genito-urinary disorders, traumatic affections, poisoning and other consequences of external origins are the most predominant diseases treated with plants. The fidelity levels of the first five species used to treat these groups of diseases range between 50 and $95.5 \%$. These diseases also belong to the most morbid ones in Madagascar.

Conclusions: The present study highlight the actual state of Madagascan medicinal plants and the corresponding ailments. These data are useful to direct the future projects of biodiversity conservation in Madagascar and pharmacological and ethnobotanical investigations of medicinal plants.
Key words: Madagascar, medicinal plants, actual state, most diversified and cited species

\section{Contexte}

Madagascar est une île de $587000 \mathrm{~km}^{2}$ de l'Océan Indien. Elle est séparée du continent africain par le canal de Mozambique, à une distance de plus de $400 \mathrm{~km}$ (Donque, 1973), et est située entre le $12^{\circ}$ et le $26^{\circ}$ de latitude Sud, traversée au Sud par le Tropique du Capricorne.

La topographie consiste en un ensemble dissymétrique, à pente raide avec de grands escarpements rectilignes, de collines et une série de petites plaines littorales étroites bordées de lagunes vers l'Est. Pourtant, vers l'Ouest, elle présente des pentes plus douces avec des abaissements par paliers des hautes terres jusqu'à des vastes plaines d'altitudes (Mangindrano, Antananarivo, Alaotra), puis des bas plateaux terminés par de larges baies ou de delta. Du Nord au Sud, dans sa partie médiane, émergent quatre principaux massifs formant la haute terre, à savoir le massif de Tsaratanàna culminant à 2876m (Maromokotra), le massif de Marojejy à $2137 \mathrm{~m}$, le massif d'Ankaratra à $2643 \mathrm{~m}$ (Tsiafajavona), et celui d'Andringitra à 2658m (pic Boby) (Begué 1966).

Madagascar est soumis à deux vents dominants. Les alizés, venant de sud-est donnent naissance à de fortes précipitations sur le versant oriental pendant toute l'année. La mousson, du secteur nordouest, apporte des pluies estivales de la partie occidentale et septentrionale.

L'altitude et l'exposition aux vents de l'alizé et de la mousson, engendrent une variété de microclimats. Ces variations microclimatiques sont à l'origine de divers types de formations végétales dont les plus dominants sont les forêts humides des côtes orientales, les forêts sèches caducifoliées de l'ouest, les forêts épineuses du sud, les forêts sclérophylles du centre, et les végétations sur des rochers des massifs centraux. Ces végétations abritent une diversité animale et végétale spectaculaires. De ce fait, la grande île constitue un des points chauds du monde (Mittermeier et al. 2004). Sa flore compte 11 220 espèces de plantes vasculaires (Callmander et al. 2011)

A ce cadre géographique, extrêmement diversifié, se sont implantés 23000000 habitants appartenant à 18 groupes ethniques. Plus de $80 \%$ de ces populations sont des ruraux et vivent aux dépens des ressources naturelles. Les activités humaines telles que les brûlis de forêts, les feux de brousses 
et les exploitations minières font dégrader chaque année 120731 ha de formations forestières par an entre 2005 et 2013 (MEEFM 2015). Nombreuses espèces végétales et animales sont ainsi menacées de disparition.

Les plantes médicinales n'échappent pas à ces menaces. A ces pressions d'origine anthropique s'ajoutent l'abandon progressif de la culture locale (Rakotoarisoa et al. 1986) et le vieillissement des guérisseurs (Bussmann 2013, De Beer \& Van Wyk 2011). Cependant, les plantes médicinales occupent une place prépondérante dans le système de soins de santé dans les pays en voie de développement où environ $80 \%$ de la population y ont recours pour se soigner (OMS 2013). Les pouvoirs curatifs des plantes dont l'efficacité est prouvée au fil des temps doivent aux principes actifs qu'elles contiennent. Nombreuses espèces médicinales sont d'utilité pharmaceutique (Havinga et al. 2010) du fait de ses principes actifs susceptibles de donner naissance à des médicaments contrôlés et standardisés (De Padua et al.1999).

A Madagascar, 89 espèces de plantes médicinales sont vendues dans les grands marchés de la capitale (Randriamiharisoa et al. 2015). Deux importantes sociétés pharmaceutiques, à savoir Homeopharma et Soamadina produisent des phytomédicaments tout en apportant des soutiens aux producteurs artisanaux d'huiles essentielles. Six espèces médicinales à vocation industrielle, dont Aphloia theiformis, Catharanthus roseus, Centella asiatica, Drosera madagascariensis, Pygeum africanum et Voacanga thouarsii, sont exportées à l'extérieur et rapportaient annuellement entre 1189171 \& et 1403 282 \$ (Rasoanaivo 1996, Andriantsiferana \& Rajaonson 1998).

En outre, les plantes utiles constituent un argument pour la conservation d'une zone donnée par les communautés locales (Ky et al. 2009). La connaissance de l'utilisation des plantes en médicine traditionnelle joue un important rôle dans la conservation de leurs habitats naturels (Hamilton 2004). Chez nombreux pays africains, le degré d'utilisation des espèces est un pré requis pour l'élaboration d'un plan de conservation (Kristensen 2003).

La connaissance des plantes médicinales de Madagascar reste insuffisante en raison du nombre encore restreint de publications afférentes. Heckel (1903), a publié un ouvrage intitulé «Les plantes médicinales et toxique de Madagascar avec leurs noms et leurs emplois indigènes» dans les Annales du Musé Colonial de Marseille. Randriamahefa et
Rakotozafy (1979) ont écrit un ouvrage, qui reste non publié, intitulé «Tari-dàlana ho an'ny zavamaniry fanao fanafody Malagasy» traduit littéralement en «Guide des plantes médicinales de Madagascar». Dans cet ouvrage figure la liste des plantes médicinales avec leurs utilisations suivant les groupes ethniques. Plotkin et al. (1985) ont rassemblé les plantes médicinales de Madagascar dans un rapport pour orienter les recherches sur les plantes médicinales de Madagascar. Rabesa et al. (1990) ont communiqué pendant le premier congrès sur l'ethnopharmacologie à Strasbourg l'état de lieux sur les plantes médicinales de Madagascar. L'ouvrage intitulé «Dictionnaire des noms malgaches des végétaux» a été publié par Boiteau et al. (1999). La synthèse des données relatives aux plantes médicinales de Madagascar s'avère ainsi importante au regard de ces initiatives d'inventaires.

Le présent travail concerne l'évaluation de l'état de lieux des inventaires des plantes médicinales de Madagascar afin d'identifier les espèces les plus utilisées en médecines traditionnelles ainsi que les maladies traitées avec les plantes médicinales et de déterminer l'importance des espèces dans le traitement des maladies.

\section{Matériels et méthodes}

Les informations issues de 20 ouvrages, 42 articles, 31 thèses et mémoires et 5 rapports parues depuis la colonisation jusqu'à nos jours sont rassemblées. Ces publications sont consultées dans divers sites web publiant les plantes médicinales comme fitoterapia, Journal of biology and ethnomedecine, et Journal of ethnopharmacology, auprès d'institutions œuvrant dans le domaine des plantes médicinales à Madagascar comme le Centre National d'Application de Recherches Pharmaceutiques (CNARP), le Missouri Botanical Garden (MBG), les Universités locales, ainsi que les centres de documentation comme l'Academie Malagasy et le Centre d'Information et de Documentation Scientifique et Techniques ou CIDST.

Les données ethnobotaniques contenues dans ces publications sont introduites par la suite dans Microsoft Excel en mettant les noms scientifiques de la plante dans les lignes et les autres informations sur les indications thérapeutiques, la partie de plante utilisée, la préparation et les modes d'emploi dans les colonnes.

Les noms scientifiques des espèces recensés sont actualisés en consultant les bases de données botaniques du Tropicos et African plant database. Quand un nom d'espèce est absent dans ces bases de données, les noms scientifiques sont identifiés à 
l'aide de l'herbier de référence déposé dans les herbaria locaux. Dans le cas où l'herbier de référence n'existe pas, le nom du genre a été retenu et le nom d'espèce est totalement rayé de la liste.

A ces données sur les publications s'ajoutent la distribution des espèces et la classification des maladies. La distribution phytogéographique des espèces recensées est documentée en consultant les bases de données botaniques du Tropicos, et African plant database. Les types de maladies indiquées pour chaque espèce sont classés selon la classification internationale des maladies ou CIM (OMS 2008).

Les fréquences de citation sont obtenues en calculant le pourcentage d'informateurs citant l'espèce, rapporté au nombre total d'informateurs enquêtés (Singh et al. 2012).

Le niveau de fidélité des espèces est déterminé pour quantifier leurs importances dans le traitement des groupes de maladies. Pour cela, la formule de Friedman et ses collaborateurs (1986) est adoptée. II s'agit de la proportion du rapport entre le nombre d'informateur qui suggère l'utilisation d'une espèce pour le traitement de maladie $\left(N_{P}\right)$ et le nombre total d'informateur qui cite l'espèce pour n'importe quelles maladies $\left(\mathrm{N}_{\mathrm{U}}\right)$.

Lors des calculs, les informateurs correspondent aux publications utilisées pendant la collecte des données.

\section{Résultats}

\section{Diversité et richesse de la flore médicinale}

Les espèces médicinales de Madagascar sont diverses et appartiennent à tous les grands groupes des végétaux.

\section{Au niveau espèce}

Les plantes médicinales de Madagascar enregistrées dans la base comptent 3245 espèces appartenant à 1050 genres et 203 familles. La liste est dominée par les Angiospermes dont 2866 appartiennent au groupe des Dicotylédones et 301 à celui des Monocotylédones (Tableau1). Les espèces des plantes inférieures sont moins représentées.

\section{Au niveau genre}

Les trente premiers genres les plus riches en espèces médicinales sont présentés dans le Tableau 2. La majorité de ces taxa sont composés d'espèces d'arbres ou arbustes comme Clerodendrum, Croton, Commiphora, Danais, Diospyros, Dypsis, Erythroxylum, Ficus, Macaranga, Grewia, Noronhia, Phyllanthus, Psorospermum,
Psychotria et Vepris. Les espèces de ces genres appartiennent surtout à des formations forestières. Les espèces des autres genres comme Aloe, Cyperus, Helichrysum, Hibiscus, Indigofera, Kalanchoe, Senecio et Vernonia sont rencontrées aussi bien dans les formations forestières que dans les formations herbeuses.

Tableau 1. Synoptique des espèces médicinales de Madagascar

Table1. Synopsis of medicinal plants from Madagascar

\begin{tabular}{|l|c|c|c|}
\hline & Espèces & Genres & Familles \\
\hline DICOTYLEDONES & 2866 & 880 & 148 \\
\hline MONOCOTYLEDONES & 301 & 123 & 27 \\
\hline PTERIDOPHYTES & 64 & 37 & 20 \\
\hline LICHENS & 7 & 3 & 2 \\
\hline GYMNOSPERMES & 4 & 4 & 3 \\
\hline ALGUES & 3 & 3 & 3 \\
\hline Total & $\mathbf{3 2 4 5}$ & $\mathbf{1 0 5 0}$ & $\mathbf{2 0 3}$ \\
\hline
\end{tabular}

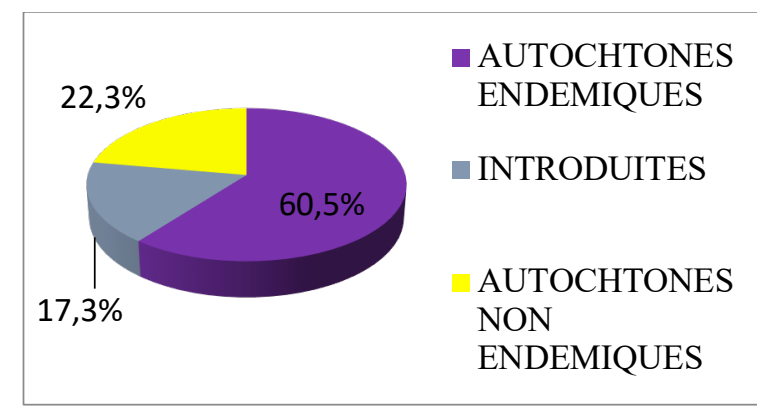

Figure 1. Endémisme des plantes médicinales de Madagascar

Figure 1. Endemism of medicinal plants from Madagascar

\section{Au niveau famille}

Les 30 premières familles les plus riches en espèces sont présentées dans le Tableau 3. Trois familles dont les Asteraceae, les Fabaceae et les Rubiaceae contiennent chacune plus de 200 espèces médicinales. Les Apocynaceae, les Euphorbiaceae, les Lamiaceae et les Malvaceae renferment des espèces dont le nombre est compris entre 100 et 200 . Vingt-trois autres familles sont représentées par moins de 100 espèces. 
Tableau 2. Trente premiers genres les plus riches en espèces médicinales

Table 2. Top 30 species-rich genera of medicinal plants

\begin{tabular}{|c|c|}
\hline Genre & $\begin{array}{l}\text { Nombre } \\
\text { d'espèces } \\
\text { médicinales }\end{array}$ \\
\hline Croton L. & 98 \\
\hline Helichrysum Mill. & 46 \\
\hline Noronhia Stadtm. ex Thouars & 39 \\
\hline Psychotria L. & 29 \\
\hline Euphorbia L. & 26 \\
\hline Aloe L. & 25 \\
\hline Crotalaria L. & 25 \\
\hline Kalanchoe Adans. & 25 \\
\hline Combretum Loefl. & 22 \\
\hline Desmodium Desv. & 22 \\
\hline Erythroxylum P. Browne & 22 \\
\hline Senecio L. & 22 \\
\hline Commiphora Jacq. & 21 \\
\hline Cynanchum L. & 21 \\
\hline Danais Comm. ex Vent. & 21 \\
\hline Indigofera L. & 21 \\
\hline Phyllanthus L. & 21 \\
\hline Ficus L. & 20 \\
\hline Diospyros L. & 20 \\
\hline Clerodendrum $\mathrm{L}$. & 19 \\
\hline Grewia L. & 19 \\
\hline Paederia L. & 19 \\
\hline Vernonia Schreb. & 18 \\
\hline Cyperus L. & 17 \\
\hline Macaranga Thouars & 17 \\
\hline Psorospermum Spach & 17 \\
\hline Vepris Comm. ex A. Juss. & 16 \\
\hline Hibiscus L. & 16 \\
\hline Impatiens L. & 16 \\
\hline Dypsis Noronha ex Mart. & 16 \\
\hline
\end{tabular}

Endémisme de la flore médicinale

Les espèces médicinales endémiques sont dominantes (Figure 1), soit plus de $60 \%$ des espèces totales. Celles non endémiques représentent $39,6 \%$ dont $22,3 \%$ partagées avec d'autres régions du monde, et $17,3 \%$ sont introduites.

Le groupe des Dicotylédones est le plus riche en espèces endémiques, soit $63,7 \%$ (Tableau 4). Les espèces appartenant au groupe des Monocotylédones ne représentent que 44\%. Quant aux Ptéridophytes, aucune espèce médicinale introduite n'est enregistrée mais ce sont surtout des espèces non endémiques (83,9\%). Par contre, toutes les Gymnospermes médicinales sont des plantes introduites.

Tableau 3. Trente premières familles les plus riches en espèces médicinales

Table 3. Top 30 species-rich families of medicinal plants

\begin{tabular}{|c|c|}
\hline Famille & $\begin{array}{l}\text { Nombre } \\
\text { d'espèces } \\
\text { médicinales }\end{array}$ \\
\hline FABACEAE & 279 \\
\hline ASTERACEAE & 238 \\
\hline RUBIACEAE & 218 \\
\hline EUPHORBIACEAE & 193 \\
\hline APOCYNACEAE & 114 \\
\hline MALVACEAE & 107 \\
\hline LAMIACEAE & 100 \\
\hline POACEAE & 63 \\
\hline RUTACEAE & 57 \\
\hline ANNONACEAE & 54 \\
\hline SOLANACEAE & 48 \\
\hline ACANTHACEAE & 44 \\
\hline MYRTACEAE & 42 \\
\hline PHYLLANTHACEAE & 42 \\
\hline LAURACEAE & 40 \\
\hline MELASTOMATACEAE & 38 \\
\hline COMBRETACEAE & 36 \\
\hline SALICACEAE & 36 \\
\hline CYPERACEAE & 35 \\
\hline SAPINDACEAE & 34 \\
\hline MELIACEAE & 33 \\
\hline CELASTRACEAE & 32 \\
\hline XANTHORRHOEACEAE & 27 \\
\hline PRIMULACEAE & 30 \\
\hline ORCHIDACEAE & 29 \\
\hline CLUSIACEAE & 24 \\
\hline ARECACEAE & 28 \\
\hline BURSERACEAE & 28 \\
\hline CONVOLVULACEAE & 27 \\
\hline CRASSULACEAE & 26 \\
\hline
\end{tabular}


Tableau 4. Endémisme des plantes médicinales à l'intérieur des grands groupes de plantes

Table 4. Endemism of medicinal plant within high classification rank

\begin{tabular}{|l|c|c|c|c|}
\hline & $\begin{array}{l}\text { Dicotylédones } \\
(\%)\end{array}$ & $\begin{array}{l}\text { Monocotylédones } \\
(\%)\end{array}$ & $\begin{array}{l}\text { Ptéridophytes } \\
(\%)\end{array}$ & $\begin{array}{l}\text { Gymnospermes } \\
(\%)\end{array}$ \\
\hline $\begin{array}{l}\text { Espèces autochtones } \\
\text { endémiques }\end{array}$ & 63,7 & 44 & 16,1 & 0 \\
\hline $\begin{array}{l}\text { Espèces autochtones } \\
\text { non endémiques }\end{array}$ & 20 & 30,4 & 83,9 & 0 \\
\hline Espèces introduites & 16,3 & 25,6 & 0,0 & 100 \\
\hline
\end{tabular}

Différents groupes de maladies traitées avec les plantes médicinales

Selon la CIM, 19 groupes de maladies sont traités avec les espèces médicinales recensées. L'histogramme présente la fréquence de citation de ces groupes de maladies (Figure 2).

Cet histogramme montre que les dix premières maladies les plus citées possèdent des fréquences de citation supérieures à $5 \%$. Ce sont les maladies de l'appareil digestif (MAD, 291 maladies), les maladies de l'appareil génito-urinaire (MAG, 135 maladies), les lésions traumatiques, empoisonnements et certaines autres conséquences de causes externes (LTE, 187 maladies), certaines maladies infectieuses et parasitaires (MIP, 87 maladies), les maladies de l'appareil respiratoire (MAR, 96 maladies), les maladies du système nerveux (MSN, 157 maladies), les maladies de la peau et du tissu cellulaire souscutané (MPT, 202 maladies), et les symptômes, signes et résultats anormaux d'examens cliniques et de laboratoire, non classés ailleurs (SYMP, 180 maladies)

Les fréquences de citation de la grossesse, accouchement et puerpéralité (GAP, 173 maladies), les maladies du système ostéo-articulaire, des muscles et du tissus conjonctif (SOA, 43 maladies), inclassables (INC, 178 maladies), les maladies endocriniennes, nutritionnelles et métaboliques (MEN, 68 maladies), les maladies de l'appareil circulatoire (MAC, 54 maladies) et maladies de l'œil et de ses annexes (OEA, 21 maladies) sont comprises entre 5 et $1 \%$. Les moins citées (fréquence inférieure à $1 \%$ ) sont les maladies du sang et des organes hématopoïétiques et certains troubles du système immunitaire (MSO), les maladies de l'oreille et de l'apophyse mastoïde (ORA, 25 maladies), les troubles mentaux et du comportement (TMC, 23 maladies), les tumeurs (TUM, 20 maladies), et certaines affections dont l'origine se situe dans la période périnatale (APP, 4 maladies).
Taxa les plus cités en médecine traditionnelle Au niveau espèce

Les 30 premières espèces les plus citées sont classées par ordre décroissant des valeurs de leur fréquence de citation (Tableau 5). Les fréquences de citation les plus élevées appartiennent à Psiadia altissima $(36,4 \%)$ et Cinnamosma macrocarpa $(32,3 \%)$ qui sont des espèces endémiques, suivi par trois espèces autochtones non endémiques, Harungana madagascariensis, Trema orientalis et Lygodium lanceolatum, dont les fréquences de citations sont respectivement $31,3 \%, 29,3 \%$ et $28,3 \%$. La première espèce introduite, Lantana camara, apparait à la sixième place.

\section{Au niveau genre}

Au niveau genre, les 30 premiers genres les plus fréquents sont classés par ordre décroissant de la valeur de leur fréquence de citation (Tableau 6). Albizia (23,5\%), Aloe (22,7\%), Aphloia (22,7\%), Catharanthus (26,1\%), Cinnamosma (26,1\%) viennent à la tête de la liste. Quatre genres mono spécifiques à Madagascar, à savoir Aphloia, Harungana, Tamarindus, et Trema, et deux représentants des genres introduits à Madagascar, Psidium et Ocimum, y figurent également. La majorité appartient à des genres avec des espèces forestières comme Albizia, Aphloia, Cinnamosma, Croton, Distephanus, Erythroxylum, Ficus, Harungana, Phyllanthus, Psorospermum et Trema. D'autres genres ont des espèces savanicoles comme Aloe, Catharanthus, Crotalaria, Desmodium, Emilia, Helichrysum, Senecio et Sida.

\section{Au niveau famille}

Au niveau famille, les 30 premières familles les plus fréquentes sont classées par ordre décroissant des valeurs de leur fréquence de citation (Tableau 7). Les familles de plantes les plus riches en espèces à Madagascar viennent toujours à la tête de la liste. Ce sont les Asteraceae $(78,2 \%)$, les Apocynaceae $(65,6 \%)$, Euphorbiaceae $(56,3 \%)$, les Fabaceae $(72,3 \%)$, les Lamiaceae $(52,9 \%)$, et les Rubiaceae $(66,4 \%)$. Cependant, des familles de plantes moins diversifiées en espèces comme les Amaranthaceae 
$(37,8 \%)$, les Apiaceae $(32,8 \%)$, les Menispermaceae $(31,1 \%)$ et les Zingiberaceae $(32,8 \%)$ figurent également parmi les plus citées.

\section{Niveau de fidélité}

Les niveaux de fidélité des 5 premières espèces par maladies sont présentés dans le tableau 8 . Le niveau de fidélité le plus élevé est attribué à Hubertia faujasiodes $(95,5 \%)$, indiquée pour les soins de Maladies de l'appareil génito-urinaire. Seules les espèces destinées au traitement des Lésions traumatiques, empoisonnements et certaines autres conséquences de causes externes (Emilia citrina, Eleusine indica, Ethulia conyzoides, Psorospermum androsaemifolium, Sigesbeckia orientalis), des Maladies de l'appareil digestif (Anthocleista madagascariensis, Cajanus cajan, Cinnamosma fragrans, Psidium guajava, Tachiadenus longiflorus), des Maladies de l'appareil génito-urinaires (Brachylaena ramiflora, Distephanus glutinosus, Hubertia faujasioides, Phyllarthron bojeranum, Woodfordia fruticosa) et des Maladies de l'appareil respiratoire (Abelmoschus esculentus, Abrus precatorius, Drosera madagascariensis, Paramollugo nudicaulis, Vernonia pectoralis) possèdent toutes des niveaux de fidélité supérieurs à $50 \%$. Les espèces servant aux soins des Maladies du sang et des organes hématopoiétiques et certains troubles du système immunitaire, des Troubles mentaux et du comportement ne dépassent pas $50 \%$.

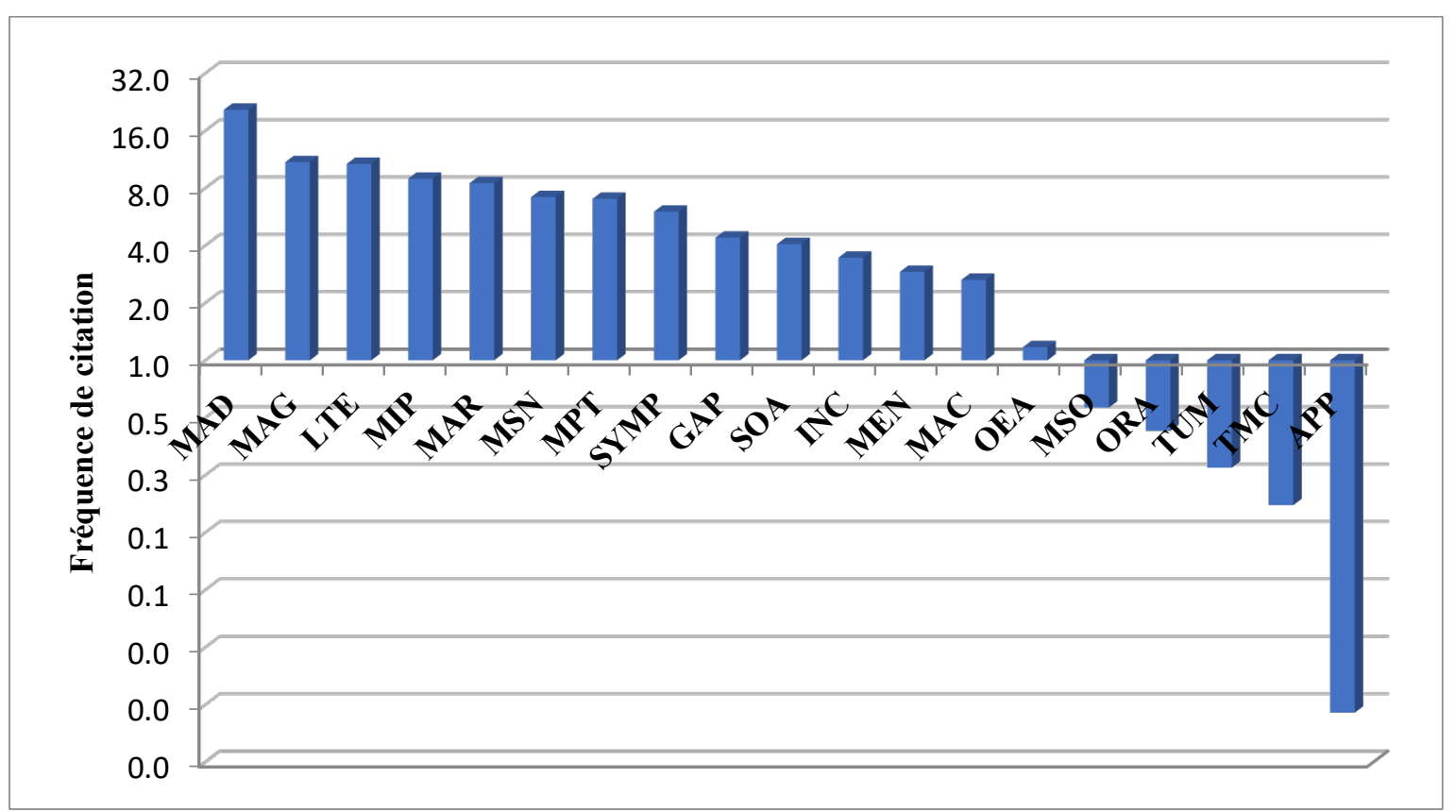

Figure 2: Maladies traitées avec les plantes médicinales (axe vertical utilisant une échelle logarithmique) Figure 2: Diseases treated with medicinal plants (vertical axes in logarithmic scale)

MAD: maladie de l'appareil digestif, MAG: maladie de l'appareil génito- urinaire, LTE: les lésions traumatiques, empoisonnements et certaines autres conséquences de causes externes, MIP : certaines maladies infectieuses et parasitaires, MAR : maladies de l'appareil respiratoire, MSN: maladies du système nerveux, MPT: maladies de la peau et du tissu cellulaire sous-cutané, SYMP: symptômes, signes et résultats anormaux d'examens cliniques et de laboratoire, non classés ailleurs, GAP: grossesse, accouchement et puerpéralité, SOA: maladies du système ostéo-articulaire, des muscles et du tissus conjonctif, INC: inclassables, MEN: maladies endocriniennes, nutritionnelles et métaboliques, MAC: maladies de l'appareil circulatoire, OEA: maladies de l'œil et de ses annexes, MSO: maladies du sang et des organes hématopoïétiques et certains troubles du système immunitaire, ORA: maladies de l'oreille et de l'apophyse mastoïde, TMC: troubles mentaux et du comportement, TUM tumeurs, APP: certaines affections dont l'origine se situe dans la période périnatale.

\section{Discussions}

\section{Diversité de plantes médicinales}

Le nombre actuel de plantes médicinales de Madagascar recensées est de 3245 espèces, soit $28,9 \%$ de la flore de Madagascar publiée par Callmander et ses collaborateurs (2011). Cette richesse reste encore inférieure à celle de Burkina Faso où les espèces médicinales représentent le tiers de sa flore (Zizka et al. 2015). La présente évaluation a permis d'adjoindre aux travaux de Rabesa et al. en 1990, un ensemble de 971 autres espèces. Ces auteurs ont publié 2274 espèces 
réparties en 808 genres et 196 familles. En outre, d'autres auteurs estiment le nombre des plantes médicinales de Madagascar à 3500 espèces (Ratsimamanga et Rasoanaivo 2005).

Tableau 5. Trente premières espèces les plus citées Table 5. Top 30 most cited species

\begin{tabular}{|c|c|}
\hline Noms scientifiques & $\begin{array}{l}\text { Fréquence } \\
\text { de citation } \\
\text { ou FC (\%) }\end{array}$ \\
\hline \begin{tabular}{|l} 
Psiadia altissima (DC.) Drake \\
\end{tabular} & 36,4 \\
\hline $\begin{array}{l}\text { Cinnamosma macrocarpa } \mathrm{H} \text {. } \\
\text { Perrier }\end{array}$ & 32,3 \\
\hline $\begin{array}{l}\text { Harungana madagascariensis } \\
\text { Lam. ex Poir. }\end{array}$ & 31,3 \\
\hline Trema orientalis (L.) Blume & 29,3 \\
\hline Lygodium lanceolatum Desv. & 28,3 \\
\hline Lantana camara L. & 27,3 \\
\hline Ageratum conyzoides L. & 26,3 \\
\hline Aphloia theiformis (Vahl.) Benn. & 26,3 \\
\hline Ocimum gratissimum $\mathrm{L}$. & 26,3 \\
\hline Catharanthus roseus (L.) G. Don & 25,3 \\
\hline Chenopodium ambrosioides L. & 25,3 \\
\hline Senna occidentalis (L.) Link & 25,3 \\
\hline Centella asiatica (L.) Urb. & 23,2 \\
\hline Abrus precatorius L. & 22,2 \\
\hline Cinnamosma fragransBaill. & 22,2 \\
\hline $\begin{array}{l}\text { Brachylaena ramiflora (DC.) } \\
\text { Humbert }\end{array}$ & 21,2 \\
\hline Carica papaya L. & 21,2 \\
\hline $\begin{array}{l}\text { Combretum coccineum }(\mathrm{H} . \\
\text { Perrier) Jonkind }\end{array}$ & 21,2 \\
\hline $\begin{array}{l}\text { Helichrysum faradifani Scott- } \\
\text { Elliot }\end{array}$ & 20,2 \\
\hline Cajanus cajan (L.) Huth & 19,2 \\
\hline Clidemia hirta (L.) D. Don & 19,2 \\
\hline Canarium madagascariense Engl. & 18,2 \\
\hline Acmella caulirhiza Delile & 17,2 \\
\hline $\begin{array}{l}\text { Aframomum angustifolium } \mathrm{K} \text {. } \\
\text { Schum. }\end{array}$ & 17,2 \\
\hline Bidens pilosa L. & 17,2 \\
\hline Buddleja madagascariensis Lam. & 17,2 \\
\hline $\begin{array}{l}\text { Anthocleista madagascariensis } \\
\text { Baker }\end{array}$ & 16,2 \\
\hline Aristolochia albida Duch. & 16,2 \\
\hline Baronia taratana Baker & 16,2 \\
\hline Burasaia madagascariensis DC. & 16,2 \\
\hline
\end{tabular}

Tableau 6. Trente premiers genres les plus cités Table 6: Top 30 most cited genera

\begin{tabular}{|c|c|}
\hline Genres & $\begin{array}{l}\text { Fréquence } \\
\text { de citation } \\
\text { ou FC (\%) }\end{array}$ \\
\hline Albizia Durazz. & 23,5 \\
\hline Aloe L. & 22,7 \\
\hline Aphloia (DC.) Benn. & 22,7 \\
\hline Catharanthus G. Don & 26,1 \\
\hline Cinnamosma Baill. & 26,1 \\
\hline Combretum Loefl. & 28,6 \\
\hline Crotalaria L. & 22,7 \\
\hline Croton L. & 30,3 \\
\hline Desmodium Desv. & 26,1 \\
\hline Distephanus Cass. & 24,4 \\
\hline Emilia Cass. & 23,5 \\
\hline Erythroxylum P.Browne & 22,7 \\
\hline Euphorbia Juss. & 32,8 \\
\hline Ficus L. & 43,7 \\
\hline Harungana Lam. & 26,9 \\
\hline Helichrysum Mill. & 42,0 \\
\hline Kalanchoe Adans. & 26,9 \\
\hline Ocimum L. & 26,1 \\
\hline Phyllanthus L. & 33,6 \\
\hline Psiadia Jacq. & 36,1 \\
\hline Psidium L. & 28,6 \\
\hline Psorospermum Spach & 23,5 \\
\hline Senecio L. & 24,4 \\
\hline Senna Mill. & 27,7 \\
\hline Sida L. & 25,2 \\
\hline Solanum L. & 28,6 \\
\hline Syzygium Steud. & 23,5 \\
\hline Tamarindus L. & 23,5 \\
\hline Trema Lour. & 26,9 \\
\hline Vernonia Schreb. & 25,2 \\
\hline
\end{tabular}

Cette différence d'effectif avec le présent résultat pourrait être due à l'actualisation des noms scientifiques apportée dans les données issues des publications. En effet, lors de la synthèse des données, certain nombre de noms scientifiques non valides et de plantes correspondant aux noms scientifiques absents à Madagascar ne possédant 
pas d'herbiers de référence déposés dans les herbaria ont été éliminées de la liste.

Tableau 7. Trente premières familles les plus citées Table 7. Top 30 most cited families

\begin{tabular}{|c|c|}
\hline Familles & $\begin{array}{l}\text { Fréquence de } \\
\text { citation ou FC } \\
(\%)\end{array}$ \\
\hline ASTERACEAE & 78,2 \\
\hline FABACEAE & 72,3 \\
\hline RUBIACEAE & 66,4 \\
\hline APOCYNACEAE & 65,6 \\
\hline EUPHORBIACEAE & 56,3 \\
\hline LAMIACEAE & 52,9 \\
\hline MORACEAE & 49,6 \\
\hline RUTACEAE & 49,6 \\
\hline PHYLLANTHACEAE & 47,1 \\
\hline MALVACEAE & 46,2 \\
\hline MYRTACEAE & 44,5 \\
\hline POACEAE & 43,7 \\
\hline SAPINDACEAE & 42,0 \\
\hline ANACARDIACEAE & 40,3 \\
\hline ANNONACEAE & 39,5 \\
\hline MELASTOMATACEAE & 38,7 \\
\hline AMARANTHACEAE & 37,8 \\
\hline COMBRETACEAE & 37,8 \\
\hline GENTIANACEAE & 37,8 \\
\hline HYPERICACEAE & 37,0 \\
\hline CELASTRACEAE & 33,6 \\
\hline ACANTHACEAE & 32,8 \\
\hline APIACEAE & 32,8 \\
\hline XANTHORRHOEACEAE & 32,8 \\
\hline BIGNONIACEAE & 32,8 \\
\hline MELIACEAE & 32,8 \\
\hline ZINGIBERACEAE & 32,8 \\
\hline LAURACEAE & 31,9 \\
\hline SOLANACEAE & 31,9 \\
\hline MENISPERMACEAE & 31,1 \\
\hline
\end{tabular}

Dans la liste, les Angiospermes et les Ptéridophytes sont dominants car ces groupes ont les plus riches en espèces de la flore de Madagascar. En effet, les Angiospermes occupent $95 \%$ de la flore malgache (Callmander et al. 2011), et les Ptéridophytes comptent 586 espèces (Rakotondrainibe 2009). Néanmoins, ces dernières sont peu connues car seulement 64 espèces ont de vertus médicinales.

\section{Endémisme}

Quant à l'endémisme, une inversion de proportion est constatée en comparant aux travaux de Rabesa et al. (1990). Ces auteurs ont publié que les espèces médicinales endémiques occupent $40 \%$ de la flore alors que dans le présent travail, elles sont de $60 \%$. L'augmentation des espèces endémiques est due aux travaux d'enquêtes menées dans les domaines forestiers où la richesse en espèces endémiques est élevée. La majorité des enquêtes effectuées avant 1982 à Madagascar suivait des grands axes routiers et des endroits accessibles (Andriatsiferana et al. 1982). Après cette date, les travaux d'inventaires faits par CNARP et la Mention de Biologie et Ecologie Végétales, en collaboration avec le Missouri Botanical Garden sont menés dans les domaines forestiers et aires protégées. Parmi les plus importantes données, ont été notés les travaux dans les régions Ambongo et Boina (Rakotobe et al. 1991), dans les forêts classées d'AnkaranaFarafangana, dans les aires protégées de Zahamena (Rakotonandrasana 2013), dans les formations forestières du complexe Baie de RignyAntsiranana (Rakotonandrasana et al. 2017), dans les nouvelles aires protégées d'Ambalabe, d'Agnalazaha, et d'Analavelona (Rakotoarivelo et al. 2015, Randrianarivony et al. 2016, Razafindraibe et al. 2013). En outre, les enquêtes effectuées auprès des tradipraticiens mettent en exergue les espèces autochtones, surtout endémiques.

Taxa les plus riches en plantes médicinales et les plus citées en médecine traditionnelle

Au niveau des familles, d'abord, la comparaison des trente premières familles les plus diversifiées de la flore (Gautier et al. 2012) avec les 30 premières familles les plus riches en espèces médicinales montre une ressemblance élevée, soit $80 \%$ ou 24 familles.

Ensuite, cette ressemblance est de $56 \%$, soit 17 espèces, si la comparaison se fait avec les trente premières familles les plus diversifiées de la flore avec celles des plus citées en médicine traditionnelles. La coïncidence de la majorité des familles les plus riches en plantes médicinales aux familles les mieux représentées de la flore est en accord avec des travaux menés aux alentours des aires protégées de Zahamena (Rakotonandrasana 2013), autour de la baie de Rigny Antsiranana (Rakotonandrasana et al. 2017) et ceux qui ont été effectués en Afrique (Nzuki Bakwaye et al. 2013). 
Les six familles les plus diversifiées de la flore ne figurant pas parmi les familles les plus diversifiées en plantes médicinales sont les Balsaminaceae, les Bignoniaceae, les Ebenaceae, les Malpighiaceae, les Pandanaceae, et les Sapotaceae.

Enfin, les familles de plantes les plus riches ou les plus citées en espèces médicinales mais qui ne sont pas parmi les plus riches en flore sont soient des familles de plantes d'intérêts alimentaires, soient des plantes riches en métabolites secondaires, soient les deux à la fois. Ce sont les Amaranthaceae, les Anacardiaceae, les Annonaceae, les Apiaceae, les Burseraceae, les Clusiaceae, les Combretaceae, les Crassulaceae, les Gentianaceae, les Hypericaceae, les Menispermaceae, les Moraceae, les Myrtaceae, les Solanaceae et les Zingiberaceae. Par exemple, les Apiaceae sont à la fois médicinales et alimentaires. Cette famille est riche en métabolites secondaires tels les alcaloïdes, les flavonoïdes, les coumarines, les terpènes, et les polyacétylènes (van Wyk et al. 2013). Pour le cas des Menispermaceae, elle est riche en alcaloïdes (Barbosa-Filho et al. 2000).

Néanmoins, au sein des familles médicinales les plus représentées, les espèces déjà connues à usage médicinal ne dépassent pas $50 \%$ des espèces dans ces familles. De plus, les espèces médicinales sont très peu représentées aussi bien dans l'ensemble de la flore que dans certains grands groupes taxonomiques comme les Ptéridophytes.

Au niveau générique, les six premiers genres les plus diversifiés en médecine traditionnelle figurent parmi les 30 premiers genres les plus diversifiés de la flore. En outre, les genres les plus riches en espèces médicinales sont généralement riches en espèces endémiques. A titre d'exemples à Madagascar, le genre Helichrysum possède 112 espèces dont deux ne sont pas endémiques, Helichrysum leucosphaerum Baker et Helichrysum triplinerve DC. (Gautier et al. 2013); le genre Noronhia est représenté par 83 espèces dont une non endémique, Noronhia cordifolia (Labat, $M$. Pignal \& O. Pascal) Hong-Wa \& Besnard existe aux Seychelles et Comores (Hong wa 2017) ; le genre Croton comprend 113 espèces endémiques et une, Croton adenophorus Baill., partagée entre les Comores et Mayotte (Berry et al.2017); les Aloe sont au nombre de 118 espèces qui sont toutes endémiques (Letsara et al. 2012).

L'appartenance des familles médicinales aux familles les plus représentées de la flore, la dominance des genres strictement forestiers et/ou riches en espèces endémiques, et la dominance des espèces médicinales endémiques montrent l'importance des formations végétales non modifiées dans la médecine traditionnelle Malagasy. Celle-ci est confirmée d'abord, par les inventaires menés dans les aires protégées de Zahamena et la pharmacopée de l'Alaotra où les espèces endémiques sont respectivement de $76,9 \%$ et $33,7 \%$ (Rakotonandrasana 2013). Ensuite, Lyon et Hardesty (2012) ont prouvé dans leurs travaux réalisés dans la Région Anosy, que les tradipraticiens prescrivent surtout les espèces endémiques. Et enfin, Bussmann (2013) a confirmé dans son travail effectué en Afrique de l'Est (Kenya et Ethiopie) que les spécialistes ont plus de connaissances en plantes médicinales que les non spécialistes. En outre, les connaissances des tradipraticiens sur les plantes médicinales ainsi que leurs modes d'utilisation sont héritées de leurs ancêtres (Rakotonandrasana 2017) ayant vécu autrefois en relation étroite avec la nature.

\section{Maladies traitées avec les plantes médicinales}

Les maladies les plus traitées avec les plantes sont diverses. Le nombre élevé de plantes médicinales utilisées pour traiter des maladies indique l'importance de ces dernières dans un pays (Zizka et al. 2015). Ainsi, les 5 premiers maladies du présent travail coïncident aux sept maladies les plus morbides à Madagascar (MINSAN 2015) qui sont, par ordre décroissant, les infections respiratoires aiguës, les affections digestives, le paludisme et parasitoses intestinales, les diarrhées et dysenteries, les affections cutanées, l'écoulement génital et ulcération génitale, et le traumatisme. De même, les maladies traitées avec les espèces ayant des niveaux de fidélité les plus élevés (dont les 5 premières, supérieur à $50 \%$ Maladies de l'appareil génito-urinaire, Lésions traumatiques, empoisonnements et certaines autres conséquences de causes externes, Maladies de l'appareil digestif, des Maladies de l'appareil génitourinaires, Maladies de l'appareil respiratoire), appartiennent également aux maladies les plus morbides à Madagascar.

En outre, ces maladies prennent d'ampleur non seulement en Afrique, mais aussi dans le monde. Les maladies de l'appareil digestif et l'infection respiratoire sont celles qui affectent le plus les enfants (Ahmadipour et al. 2016), surtout dans beaucoup de pays en Afrique. Le paludisme, les diarrhées et les dysenteries constituent des importantes maladies humaines (Havinga et al. 2010).

Les maladies de la peau sont présentes dans le monde entier et représentent environ 34\% de toutes les maladies rencontrées (Abassi et al. 2010). Elles 
sont difficiles à traiter et ont d'impact significatif sur la qualité de la vie humaine (De wet et al. 2013). Concernant les maladies de l'appareil uro-génital, les infections sexuellement transmissibles ont de profondes répercussions sur la santé et la vie des enfants, des adolescents et des adultes partout dans le monde (OMS, 2016).

Tableau 8. Niveau de fidélité des 5 premières espèces par maladies $\left(\mathbf{N}_{\mathrm{p}}\right.$ : nombre d'informateur qui suggère l'utilisation d'une espèce pour le traitement de maladie, $\mathbf{N}_{\mathrm{U}}$ : nombre total d'informateur qui cite l'espèce pour n'importe quelles maladies, FL : niveau de fidélité) ; *seules les espèces dont $N_{u}$ supérieure ou égale à 5 sont présentées

Table 8. Level of fidelity of the first 5 species by disease (NP: number of informants who suggest the use of a species for the treatment of disease, NU: total number of informants who quote the species for any diseases, FL: level of fidelity); * only species with NU greater than or equal to 5 are presented.

\begin{tabular}{|c|c|c|c|c|}
\hline Espèces & $N_{P}$ & $N_{u}$ & FL & Maladies \\
\hline Chenopodium ambrosioides $\mathrm{L}$. & 23 & 25 & 92,0 & \multirow{5}{*}{$\begin{array}{l}\text { Certaines maladies } \\
\text { infectieuses et parasitaires }\end{array}$} \\
\hline Combretum coccineum (Sonn.) Lam. & 13 & 21 & 61,9 & \\
\hline Burasaia madagascariensis DC. & 8 & 16 & 50,0 & \\
\hline Maesa lanceolata Forssk. & 10 & 21 & 47,6 & \\
\hline Tamarindus indica L. & 11 & 27 & 40,7 & \\
\hline Cedrelopsis grevei Baill. & 5 & 10 & 50,0 & \multirow{5}{*}{$\begin{array}{l}\text { Grossesse, accouchement et } \\
\text { puerpéralité }\end{array}$} \\
\hline Leptadenia madagascariensis Decne & 9 & 18 & 50,0 & \\
\hline Ocimum gratissimum L. & 13 & 26 & 50,0 & \\
\hline Jatropha curcas L. & 9 & 20 & 45,0 & \\
\hline Psiadia altissima (DC.) Drake & 2 & 35 & 5,7 & \\
\hline Eleusine indica (L.) Gaertn. & 9 & 11 & 81,8 & \multirow{5}{*}{$\begin{array}{l}\text { Lésions traumatiques, } \\
\text { empoisonnements et } \\
\text { certaines autres } \\
\text { conséquences de causes } \\
\text { externes }\end{array}$} \\
\hline Ethulia conyzoides L.F. & 8 & 11 & 72,7 & \\
\hline Emilia citrina DC. & 12 & 17 & 70,6 & \\
\hline Psorospermum androsaemifolium Baker & 12 & 17 & 70,6 & \\
\hline Sigesbeckia orientalis L. & 11 & 16 & 68,8 & \\
\hline Emilia citrina DC. & 10 & 17 & 58,8 & \multirow{5}{*}{$\begin{array}{l}\text { Maladies de la peau et du } \\
\text { tissu cellulaire sous-cutané }\end{array}$} \\
\hline Harungana madagascariensis Lam. ex Poir. & 14 & 30 & 46,7 & \\
\hline Hubertia faujasioides (Baker) C. Jeffrey & 10 & 22 & 45,5 & \\
\hline Crinum firmifolium Baker & 5 & 11 & 45,5 & \\
\hline Smilax anceps Willd. & 9 & 22 & 40,9 & \\
\hline Senna alata (L.) Roxb. & 8 & 15 & 53,3 & \multirow{5}{*}{$\begin{array}{l}\text { Maladies de l'appareil } \\
\text { circulatoire }\end{array}$} \\
\hline Passiflora edulis Sims & 8 & 16 & 50,0 & \\
\hline Cajanus cajan (L.) Huth & 9 & 19 & 47,4 & \\
\hline Lantana camara L. & 11 & 26 & 42,3 & \\
\hline Catharanthus roseus (Bojer ex A. DC.) Pichon & 9 & 25 & 36,0 & \\
\hline Psidium guajava $L$. & 22 & 24 & 91,7 & \multirow{5}{*}{ Maladies de l'appareil digestif } \\
\hline Anthocleista madagascariensis Baker & 13 & 17 & 76,5 & \\
\hline Cajanus cajan (L.) Huth & 14 & 19 & 73,7 & \\
\hline Tachiadenus longiflorus Grisebach & 11 & 15 & 73,3 & \\
\hline Cinnamosma fragrans Baill. & 15 & 21 & 71,4 & \\
\hline
\end{tabular}




\begin{tabular}{|c|c|c|c|c|}
\hline Hubertia faujasiodes (Baker) C. Jeffrey & 21 & 22 & 95,5 & \multirow{4}{*}{$\begin{array}{l}\text { Maladies de l'appareil génito- } \\
\text { urinaire }\end{array}$} \\
\hline Distephanus glutinosus (DC.) H. Rob. \& B. Kahn & 13 & 16 & 81,3 & \\
\hline Phyllarthron bojeranum DC. & 13 & 20 & 65,0 & \\
\hline Brachylaena ramiflora (DC.) Humbert & 12 & 20 & 60,0 & \\
\hline Espèces & $\mathbf{N}_{\mathbf{P}}$ & $\mathbf{N}_{\mathbf{u}}$ & FL & Maladies \\
\hline Woodfordia fruticosa (L.) Kurz & 9 & 15 & 60,0 & \\
\hline Abrus precatorius L. & 19 & 21 & 90,5 & \multirow{5}{*}{$\begin{array}{l}\text { Maladies de l'appareil } \\
\text { respiratoire }\end{array}$} \\
\hline Abelmoschus esculentus (L.) Moench & 5 & 6 & 83,3 & \\
\hline Vernonia pectoralis Baker & 12 & 15 & 80,0 & \\
\hline Paramollugo nudicaulis (Scott Elliot) Thulin & 17 & 22 & 77,3 & \\
\hline Drosera madagascariensis DC. & 8 & 11 & 72,7 & \\
\hline Triumfetta rhomboidea Jacq. & 6 & 9 & 66,7 & \multirow{5}{*}{$\begin{array}{l}\text { Maladies de l'œil et de ses } \\
\text { annexes }\end{array}$} \\
\hline Ageratum conyzoides L. & 7 & 25 & 28,0 & \\
\hline Cajanus cajan (L.) Huth & 5 & 19 & 26,3 & \\
\hline Harungana madagascariensis Lam. ex Poir. & 7 & 30 & 23,3 & \\
\hline Mystroxylon aethiopicum (Thunb.) Loes. & 7 & 30 & 23,3 & \\
\hline Phragmites australis (Cav.) Trin. ex Steud. & 4 & 12 & 33,3 & \multirow{2}{*}{$\begin{array}{l}\text { Maladies de l'oreille et de } \\
\text { l'apophyse mastoïde* }\end{array}$} \\
\hline Kalanchoe prolifera (Bowie ex Hook.) Raym.-Hamet & 2 & 16 & 12,5 & \\
\hline Drosera madagascariensis DC. & 4 & 11 & 36,4 & \multirow{5}{*}{$\begin{array}{l}\text { Maladies du sang et des } \\
\text { organes hématopoïétiques et } \\
\text { certains troubles du système } \\
\text { immunitaire }\end{array}$} \\
\hline Physena madagascariensis Thouars ex Tul. & 4 & 18 & 22,2 & \\
\hline Catharanthusroseus (L.) G. Don & 5 & 25 & 20,0 & \\
\hline Senna occidentalis (L.) Link & 4 & 24 & 16,7 & \\
\hline Phragmites australis (Cav.) Trin. ex Steud. & 2 & 12 & 16,7 & \\
\hline Datura stramonium L. & 6 & 8 & 75,0 & \multirow{5}{*}{ Maladies du système nerveux } \\
\hline Mimosa pudica L. & 10 & 20 & 50,0 & \\
\hline Helichrysum gymnocephalum (DC.) Humbert & 7 & 19 & 36,8 & \\
\hline Mystroxylon aethiopicum (Thunb.) Loes. & 11 & 30 & 36,7 & \\
\hline Phyllanthus casticum P. Willemet & 8 & 24 & 33,3 & \\
\hline Neobeguea mahafaliensisJ.-F. Leroy & 8 & 9 & 88,9 & \multirow{5}{*}{$\begin{array}{l}\text { Maladies du système ostéo- } \\
\text { articulaire, des muscles et du } \\
\text { tissu conjonctif }\end{array}$} \\
\hline Woodfordia fruticosa (L.) Kurz & 9 & 15 & 60,0 & \\
\hline Kalanchoe prolifera (Bowie ex Hook.) Raym.-Hamet & 8 & 16 & 50,0 & \\
\hline Phyllarthron bojeranum DC. & 8 & 20 & 40,0 & \\
\hline Helichrysum gymnocephalum (DC.) Humbert & 6 & 19 & 31,6 & \\
\hline Anacardium occidentale L. & 7 & 14 & 50,0 & \multirow{5}{*}{$\begin{array}{l}\text { Maladies endocriniennes, } \\
\text { nutritionnelles et } \\
\text { métaboliques }\end{array}$} \\
\hline Helichrysum gymnocephalum (DC.) Humbert & 9 & 19 & 47,4 & \\
\hline Senna occidentalis (L.) Link & 10 & 24 & 41,7 & \\
\hline Curcuma longa L. & 6 & 19 & 31,6 & \\
\hline Harungana madagascariensis Lam. ex Poir. & 7 & 30 & 23,3 & \\
\hline Cedrelopsis grevei Baill. & 6 & 10 & 60,0 & \multirow{3}{*}{$\begin{array}{l}\text { Symptômes, signes et } \\
\text { résultats anormaux } \\
\text { d'examens cliniques et de }\end{array}$} \\
\hline Musa paradisiaca L. & 5 & 12 & 41,7 & \\
\hline Smilax anceps Willd. & 9 & 22 & 40,9 & \\
\hline
\end{tabular}




\begin{tabular}{|l|r|r|r|l|} 
Ocimum gratissimum L. & 10 & 26 & 38,5 & $\begin{array}{l}\text { laboratoire, non classés } \\
\text { ailleurs }\end{array}$ \\
\hline Cinnamosma fragrans Baill. & 7 & 21 & 33,3 & \\
\hline Tabernaemontana coffeoides Bojer ex A. DC. & 1 & 11 & 9,1 & $\begin{array}{l}\text { Troubles mentaux et du } \\
\text { comportement * }\end{array}$ \\
\hline
\end{tabular}

\begin{tabular}{|l|r|r|r|l|}
\hline Espèces & $\mathbf{N}_{\mathbf{P}}$ & \multicolumn{1}{l|}{$\mathbf{N}_{\mathbf{U}}$} & \multicolumn{1}{l|}{ FL } & Maladies \\
\hline Symphonia tanalensis Jum. \& H. Perrier & 3 & 5 & 60,0 & \\
\cline { 1 - 3 } Crotalaria berteroana DC. & 3 & 7 & 42,9 & \multirow{2}{*}{ Tumeurs* $^{*}$} \\
\cline { 1 - 2 } Catharanthus roseus (Bojer ex A. DC.) Pichon & 3 & 25 & 12,0 & \\
\hline
\end{tabular}

\section{Conclusion}

Les plantes médicinales de Madagascar sont diverses mais n'atteignent pas encore le tiers de sa flore. Elles comptent 3245 espèces reparties dans 1053 genres et 203 familles. Les espèces médicinales autochtones prédominent car, $60 \%$ de ces espèces sont endémiques, les deux premières espèces les plus citées sont endémiques, les deux espèces suivantes sont autochtones non endémiques, la première espèce introduite n'apparait qu'en sixième position. En comparaison des données du dernier recensement, 971 espèces sont nouvellement connues.

Quatre-vingt pourcent des familles les plus riches et soixante-cinq pourcent des familles les plus citées en médicine traditionnelle coïncident aux familles les plus riches en espèces de la flore. Des familles de plantes moins riches en espèces comprenant d'espèces alimentaires et/ou riche en métabolites secondaires figurent également parmi les espèces les plus citées.

Ces espèces prennent une place importante dans le système de soins des Malagasy. Dix-neuf maladies sont soignées avec des plantes médicinales. Les maladies les plus traitées avec les plantes correspondent aux maladies les plus morbides à Madagascar. En outre, les espèces ayant un niveau de fidélité élevé servent également à traiter ces maladies.

La base de données de ces espèces médicinales est disponible au Département Ethnobotanique et Botanique du CNARP. Cette base constitue un outil à l'élaboration d'un plan de conservation et de gestion durable des plantes médicinales pour que les futures générations puissent en tirer profit. Elle aide également à l'orientation des recherches pharmacologiques et aux futurs travaux d'enquêtes ethnobotaniques. Les investigations chimiques et biologiques contribueront à une meilleure valorisation de la diversité végétale et des savoirs traditionnelles.

\section{Déclarations}

Ethiques d'approbation et consentement de participation: Cette recherche a obtenu l'accord du Conseil Scientifique d'Orientation (une sorte de comité d'éthique) du Centre National d'Application de Recherches Pharmaceutiques en 2017 et 2018.

\section{Consentement à la publication: "Non applicable"}

Disponibilité des données et des matériaux: La base de données de plantes médicinales issue de cette activité est disponible au Département d'Ethnobotanique et de Botanique du Centre National d'Application de Recherches Pharmaceutiques (CNARP). Antananarivo. Madagascar.

Conflit d'intérêts: Les auteurs déclarent qu'ils n'ont pas de conflit d'intérêts.

Financement: Cette recherche est financée par le Ministère l'Enseignement Supérieur et de la Recherche Scientifique de Madagascar.

Contribution des auteurs: Rakotonandrasana Stéphan Richard et Rafidison Verohanitra ont fait la conception des travaux, l'élaboration de la méthodologie, le premier plan de la rédaction. Rakotondrafara Andriamalala, Rakotoarisoa Marrino et Ratsimandresy rassemblent et analysent les données. Rasamison Vincent traduit le résumé en anglais et améliore la rédaction. Tous les auteurs relisent et approuvent l'article avant la soumission.

\section{Remerciements}

Nous remercions ici tous les ethnobotanistes travaillant dans le domaine des plantes médicinales à Madagascar, contribuant à l'inventaire des plantes médicinales dans diverses régions de Madagascar. Nous exprimons notre gratitude au Ministère de l'Enseignement Supérieur et de la Recherche Scientifique d'avoir accepté et financé notre programme de recherches. 


\section{Références bibliographiques}

Ahmadipour S, Ahmadipour SH, Mohsenzadeh A, Asadi-Samani M. 2016. The importance of some native medicinal plants of Iran effective on gastrointestinal disorders in children: A review. Der Pharmacia Lettre 8(1):61-66.

Andriantsiferana R. 1982. Enquêtes ethnopharmacognosiques et grands axes des travaux du CNRP. Archives du Centre National de Recherches Pharmaceutiques (CNRP) 1:5-15.

Andriantsiferana R, Rajaonson H. 1998. Bilan des filières de la Biodiversité. Office National pour l'Environnement. Antananarivo.

Barbosa-Filho JM, Ca-Cunha EVL, Gray Al. 2000. Alkaloids of the Menispermaceae. In: The Alkaloids. Edited by GA Cordell. vol. 54. Academic Press, Illinois, Pp. 1-190.

Begué L. 1966. Chronique phytogéographique: la végétation de Madagascar. Bois et forêts des tropiques 106:56-65.

Berry PE, Kainulainen K, Van Ee B. 2017. A Nomenclator of Croton (Euphorbiaceae) in Madagascar, the Comoros Archipelago, and the Mascarene Islands. Phytokeys 90:1-87.

Bussman R. 2013. East African plant use-difference in plant use between nomadic and agricultural societies. In: Proceedings of the XIX th AETFAT Congress for African Plant Diversity, Systematics and Sustainable Development, Antananarivo.Edited by $\mathrm{N}$ Beau, $\mathrm{S}$ Dessein \& $\mathrm{E}$ Robbrecht. National Botanic Garden of Belgium, Meise 50:339-407.

Callmander MW, Phillipson PB, Schatz GE, Andriambololonera S, Rabarimanarivo $M$, Rakotonirina N, Raharimampionona J, Chatelain C, Gautier L, Lowry PP II. 2011. The endemic and nonendemic vascular flora of Madagascar updated. Plant Ecology and Evolution 144(2):121-125.

De Beer JJJ, Van Wyk B.-E. 2011. An ethnobotanical survey of the Agter-Hantam, Northern Cape Province, South Africa. South African Journal of Botany 77:741-744.

De Padua LS, Bunyapraphatsara N, Lemmens RHMJ. 1999. Medicinal and poisonous plant. Plant resources of South- East Asia 12 (1). BlackhuysPublishers Leiden, The Netherlands.

De Wet H, Nciki S, van Vuuren SF. 2013. Medicinal plants used for the treatment of various skin disorders by a rural community in northern Maputaland, South Africa. Journal of Ethnobiology and Ethnomedicine doi:10.1186/1746-4269-9-51

Donque G. 1973. Les conditions générales du climat de Madagascar. Madagascar: revue géographique 22:1-91.
Friedman J, Yaniv Z, Dafni A, Palewitch D. 1986. A preliminary classification of the healing potential of medicinal plants, based on a rational analysis of an ethnopharmacological field survey among Bedouins in the Negev Desert, Israel. Journal of Ethnopharmacology 16:275-287.

Gautier L, Chatelain C, Callmander MW, Phillipson PB. 2013. Richness, similarity and specificity of Madagascar flora compared with Sub-Saharan Africa. Plant Ecology and Evolution 145:55-64.

Hamilton AC. 2004. Medicinal plants, conservation and livelihoods. Biodiversity and Conservation 13:1477-1517.

Havinga RM, Hartl A, Putscher J, Prehsler S, Buchmann C, Vogl CR. 2010. Tamarindus indica L. (Fabaceae): patterns of use in traditional African medicine. Journal of Ethnopharmacology 127:573588.

Heckel E. 1903. Les plantes médicinales et toxiques de Madagascar avec leurs noms et leurs emplois indigènes. Institut Colonial de Marseille.

Hong Wa C. 2016. A taxonomic revision of Noronhia Stadm. Ex Thouars (Oleaceae) in Madagascar and Comoros Islands. Boissiera 68:1-210.

Ky J, Zorbo P, Gnoula C, Simpore J, Nikiema J, Millogo-Rasolodimby J. 2009. Medicinal

plants used in traditional medicine in the centre East region of Burkina Faso. Pakistan Journal of Biological Sciences 12:1287-1298.

Kristensen M, Lykke AM. 2003. Informant-based valuation of use and conservation preferences of savanna trees in Burkina Faso. Economic Botany 57:203-217.

Boiteau P, Boiteau M, Allorge-Boiteau L. 1999. Dictionnaire des noms malgaches des végétaux. Claude Alzieu, Grenoble.

Letsara R, Rakotoarisoa S, Almeda F. 2012. Three new Aloe A. Rich. species from Madagascar. Malagasy Nature 6:46-55.

Lyon LM, Hardesty LH. 2012. Quantifying medicinal plant knowledge among non-specialist Antanosy villagers in southern Madagascar. Economic Botany 66 (1):1-11.

Ministère de l'Environnement, de l'Ecologie, de la Météorologie et des Forêts (MEEMF), 2015. Changement de la couverture de forêts naturelles à Madagascar, 2005, 2010, 2013. Antananarivo.

Ministère de la Santé Publique (MINSAN). 2015. Annuaire des statistiques du secteur santé de Madagascar. Service des statistiques sanitaires et démographiques.

Mittermeier RA, Gil PR, Hoffmann M, Pilgrim J, Brooks T, Mittermeier J, Lamoreux CG, Da Fonseca 
GAB. 2004. Hots spots Revisited. Earth's biologicaly richest and most endangered terrestrial ecoregions. CEMEX.

Nzuki Bakwaye F., Termote C., Kibungu K., Van Damme P., 2013. Identification et importance locale des plantes médicinales utilisées dans la région de Mbanza-Ngungu, République Démocratique du Congo. Bois et Forêts des Tropiques 316 (2):63-77. Organisation Mondiale de la Santé. 2008. Classification Statistique Internationale des Maladies et des problèmes de santé connexes (CIM10), OMS, volume 2. Genève.

Organisation Mondiale de la Santé. 2013. Stratégie de l'OMS pour la médecine traditionnelle 2014-2023, OMS, Hong Kong.

Organisation Mondiale de la Santé. 2016. Stratégie mondiale du secteur de la santé contre les

infections sexuellement transmissibles 2016-2021.

OMS Département Santé reproductive et recherche. Genève.

Plotkin M, Randrianasolo V, Sussman L, Marshall NT. 1985. Ethnobotany in Madagascar. Overview, Action plan, Database. Rapport non publié, UICN.

Rabesa ZA, Rabenoro C, Andriantsiferana R, Rakotobe EA. 1990. Notes on Malagasy plants used in the traditional pharmacopoeia. Strasbourg, First International congress on Ethnopharmacology, June 5-9, 1990.

Rakotoarisoa JA. 1986. Principaux aspects des formes d'adaptation de la société traditionnelle Malgache Imn: Madagascar, Society and History. Edited by PC Kottak, JA Rakotoarisoa, A Southall \& $P$ Verin. Carolina Academic Press, Carolina.

Rakotoarivelo $\mathrm{NH}, \quad$ Rakotoarivony $\mathrm{F}$, Ramarosandratana AV, Jeannoda V, Kuhlman AR, Randrianasolo A, Bussmann RW. 2015. Medicinal plants used to treat the most frequent diseases encountered in Ambalabe rural community, Eastern Madagascar. Journal of Ethnobiology and Ethnomedecine. doi:10.1186/s13002-015-0050-2.

Rakotonandrasana SR. 2013. Les plantes médicinales de l'aire protégée de Zahamena (Madagascar) et de ses environs: richesse floristique et endémicité. In: Proceedings of the XIX th AETFAT Congress for African Plant Diversity, Systematics and Sustainable Development. Antananarivo. Edited by $\mathrm{N}$ Beau, $\mathrm{S}$ Dessein \& $\mathrm{E}$ Robbrecht. National Botanic Garden of Belgium, Meise 50:356-362.

Rakotondrainibe F. 2009. Diversité, écologie et distribution de la flore Ptéridologique. In Paysages naturels et biodiversité de Madagascar. Edited by SM Goodman MNHN, Paris, 141-160,

Randriamahefa M, Rakotozafy A, 1979. Tari-dalana ahafantarana ny raokandro Malagasy. Recherches
Bibliographiques sur les utilisations empiriques des plantes médicinales malagasy. Document Inédit.

Randriamiharisoa MN, Kuhlman AR, Jeannoda V, Rabarison H, Rakotoarivelo N, Randrianarivony $\mathrm{T}$, Rakotoarivony F, Randrianasolo A, Bussmann RW. 2015. Medicinal plants sold in the markets of Antananarivo, Madagascar. Journal of Ethnobiology and Ethnomedecine. doi:10.1186/s13002-015-0046y.

Randrianarivony $\mathrm{T}, \quad$ Randrianasolo A, Andriamihajarivo $\mathrm{T}$, Ramarosandratana AV, Jeannoda, VH, Rakotoarivony $F$, Bussmann R. 2016. Useful plants and tradition for pregnancy, child delivery and for post-partum care used by people living around Analavelona forest in South west Madagascar. Indian Journal of Traditional Knowledge 15(1):68-78.

Randrianasolo A, Lowry PPII, Schatz GES. 2017. Taxonomic treatment of Abrahamia Randrian. \& Lowry a new genus of Anacardiaceae from Madagascar. Boissiera 71:1-148.

Razafindraibe M, Kuhlman AR, Rabarison $\mathrm{H}$, Rakotoarimanana V, Rajeriarison C, Rakotoarivelo $\mathrm{N}$, Randrianarivony $\mathrm{T}$, Rakotoarivony $\mathrm{F}$, Ludovic $\mathrm{R}$, Randrianasolo A, Bussmann RW. 2013. Medicinal plants used by women from Agnalazaha littoral forest (Southeastern Madagascar). Journal of Ethnobiology and Ethnomedicine. doi:10.1186/17464269-9-73.

Rasoanaivo P. 1996. Plantes médicinales et aromatiques à valeurs économiques à Madagascar. Cahier du CITE, Série ${ }^{\circ} 4$, trim. Antananarivo.

Ratsimamanga S, Rasoanaivo P. 2005. Sur les plantes malgaches à activités biologiques prometteuses. Ethnopharmacologie 36:62-68.

Singh AG, Kumar A, Tewari DD. 2012. An ethnobotanical survey of medicinal plants used in Terai forest of western Nepal. Journal of Ethnobiology and Ethnomedicine. doi:10.1186/17464269-8-19.

\section{References bibliographiques consultees pour la construction de la base de donnees Bibliographical references used for the elaboration of database}

Beaujard P. 1988. Plantes et médecine traditionnelle dans le Sud-Est de Madagascar. Journal of Ethnopharmacology 23:165-265.

Boiteau P. 1974. Dictionnaire des noms malgaches de végétaux. Fitoterapia 2(XLV):67-81.

Boiteau P.1974. Dictionnaire des noms malgaches de végétaux. Fitoterapia 4(XLV):135-179.

Boiteau P. 1974. Dictionnaire des noms malgaches de végétaux. Fitoterapia 6(XLV):223-266. 
Boiteau P. 1975. Dictionnaire des noms malgaches de végétaux. Fitoterapia 1(XLVI):9-43.

Boiteau P. 1975. Dictionnaire des noms malgaches de végétaux. Fitoterapia 2(XLVI):83-95.

Boiteau P. 1975. Dictionnaire des noms malgaches de végétaux. Fitoterapia 3(XLVI):111-135.

Boiteau P. 1975. Dictionnaire des noms malgaches de végétaux. Fitoterapia 5(XLVI):201-239.

Boiteau P. 1976. Dictionnaire des noms malgaches de végétaux. Fitoterapia 3(XLVI):137-143.

Boiteau P. 1976. Dictionnaire des noms malgaches de végétaux. Fitoterapia 4(XLVI):151-191.

Boiteau P. 1977. Dictionnaire des noms malgaches de végétaux. Fitoterapia 1(XLVI) 9-47.

Boiteau P. 1977. Dictionnaire des noms malgaches de végétaux. Fitoterapia 2(XLVI):73-96.

Boiteau P. 1977. Dictionnaire des noms malgaches de végétaux. Fitoterapia 6(XLVI):279-287.

Boiteau P. 1978. Dictionnaire des noms malgaches de végétaux. Fitoterapia 1(XLIX):39-47.

Boiteau P. 1978. Dictionnaire des noms malgaches de végétaux. Fitoterapia 4(XLVI):179-192.

Boiteau P. 1978. Dictionnaire des noms malgaches de végétaux. Fitoterapia 2(XLIX):85-96.

Boiteau P. 1978. Dictionnaire des noms malgaches de végétaux. Fitoterapia 3(XLIX):111-144.

Boiteau P. 1978. Dictionnaire des noms malgaches de végétaux, Fitoterapia 5(XLVIII):231-240.

Boiteau P. 1978. Dictionnaire des noms malgaches de végétaux. Fitoterapia 6(XLIX):265-288.

Boiteau P. 1979. Dictionnaire des noms malgaches de végétaux. Fitoterapia 1(XLVIII):25-47.

Boiteau P. 1979. Dictionnaire des noms malgaches de végétaux. Fitoterapia 2(L):73-96.

Boiteau P. 1979. Dictionnaire des noms malgaches de végétaux. Fitoterapia 4(L):167-192.

Boiteau P. 1979. Dictionnaire des noms malgaches de végétaux. Fitoterapia 5(VIII):207-240.

Boiteau P.1986. Médecine traditionnelle et pharmacopée: précis de matière médicale malgache. ACCT.

Boiteau P, Boiteau M, Allorge-Boiteau L. 1999. Dictionnaire des noms malgaches de végétaux, Vol I, Alzieu. Grenoble.

Boiteau P, Boiteau M, Allorge-Boiteau L. 1999. Dictionnaire des noms malgaches de végétaux, $\mathrm{Vol}$ II, Alzieu. Grenoble.

Boiteau P, Boiteau M, Allorge-Boiteau L. 1999. Dictionnaire des noms malgaches de végétaux, Vol III, Alzieu. Grenoble.
Boiteau P, Boiteau M., Allorge-Boiteau L.1999. Dictionnaire des noms malgaches de végétaux, Vol. IV. Alzieu. Grenoble.

Bost R. 1961. Pharmacopée Malgache. Mémoires de l'institut scientifique de Madagascar, série $\mathrm{B}, \mathrm{X}(2): 161-234$.

Botsalahy JJL. 2007. Valorisation des plantes médicinales. Mémoire de Master 1, Faculté des sciences, Université de Mahajanga.

Debray M, Jacquemin H, Razafindrambao R. 1971. Contribution à l'inventaire des plantes médicinales de Madagascar, in Travaux et documents de I'ORSTOM N8, ORSTOM Paris.

Descheemacker A. 1979. Ravimaitso. Fianarantsoa. Du Puy DJ, Labat J.-N, Rabevohitra R, Villiers J.-F, Bosser J, Morat J. 2002. The Leguminosae of Madagascar, Royal Botanical Guarden, Kew.

Faranirina L. 2003. Etudes ethnobotaniques, biologiques et écologiques des plantes utiles dans la forêt d'Antsahabe Est (Anjozorobe). Mémoire de DEA, Faculté des Sciences, Université d'Antananarivo.

Gallé JB, Groeber G Ledoux A, Nicolas J.-P. 2014. Quelques plantes employées dans le Sud-Ouest de Madagascar: Ethnobotanique/Monographies scientifiques, Editions Jardins du Monde. Saint Thonan, France.

Gruca M, Blanche-Overgraad A, Dransfield J, Balsev H. 2016. Medicinal palms (Arecaceae) in Madagascar: undocumented or underutilized?, Botanical Journal of the Linnean Society 182:517525.

Hanitriniaina NF. 2018. Utilisations traditionnelles et écologie de Pimpinella perrieri Sales et Hedge et Pimpinella ebracteata Baker (Apiaceae), Mémoire de licence professionnelle en Gestion de l'Environnement, Mention Biologie, Institut d'Enseignement Supérieur AntsirabeVakinankaratra, Antsirabe.

Heckel E. 1903. Les plantes médicinales et toxiques de Madagascar avec leurs noms et leurs emplois indigènes. Institut Colonial de Marseille.

Isaia R. 1995. Approche Phytoécologique sur l'Evaluation Qualitative et Quantitative des Utilisations Villageoises des Ressources Naturelles en Forêt Dense Humide Sempervirente. Mémoire de DEA, Faculté des Sciences, Université d'Antananarivo.

Jacquemin H. 1971. Plantes médicinales de l'ile Sainte Marie. Rapport.

Kaufmann JC, Elvin-Lewis. 1995. Towards a logic of ethnodentistry at Antogobe, southern Madagascar. Economic botany 49(2):213-222. 
Laivao MO. 1995. Contribution à l'étude de la flore médicinale de Bemaraha et leurs caractéristiques écologiques. Mémoire de DEA Faculté de Sciences, Université d'Antananarivo.

Manajaniaina S. 2018. Etude préliminaire sur l'Ecologie et évaluation quantitative de l'utilisation locale de Billburttia capensoides Sales \& Hedge (Apiaceae) et Tetradenia goudotii Briq. (Lamiaceae) dans la Commune rurale Andranomiely, versant ouest du massif d'Ankaratra, Mémoire de licence professionnelle en Gestion de l'Environnement, Mention biologie, Institut d'Enseignement Supérieur Antsirabe- Vakinankaratra. Antsirabe.

Miarisoa R. 2012. Inventaire des plantes médicinales des forêts aux alentours des zones de conservation du projet Ambatovy en vue de leurs valorisations. Mémoire de fin d'études ESSA/Forêt., Université d'Antananarivo.

Nicolas JP. 2012. Plantes médicinales du Nord de Madagascar, Ethnobotanique Antakarana et informations scientifiques. Editions Jardins du monde, Saint Thonan, France.

Norodiny. 2011. Valorisation des filières potentielles en matière de biodiversité : les plantes médicinales dans l'aire protégée communautaire "Tsinjoriake", Sud-Ouest de Masagascar. Mémoire de DEA en Biodiversité et Environnement, Faculté de Science, Université de Toliara.

Norscia I, Borgognini-Tarli SM. 2006. Ethnobotanical reputation of plant species from two forests of Madagascar: A preliminary investigation (Kirindy and Sainte Luce forest).South African Journal of Botany 72:656-660.

Novy JW. 1997. Medicinal plants of the eastern region of Madagascar. Journal of Ethnopharmacology 55:119-126.

Office National pour l'Environnement, 2006. "Kit pédagogique : Région Anosy". Edition Delachaux et Niestlé S.A., Lausanne, Paris.

Onjalalaina GE. 2014. Inventaire, études ethnobotanique et écologique, statut de conservation et criblage phytochimique des plantes de l'aire protégée de Tampolo Fénérive Est (Analanjirofo). Mémoire de DEA. Université d'Antananarivo.

Pernet RB. 1957. Les plantes médicinales malgache. Mémoires de I'Institut Scientifique de Madagascar B(VII):1-144.

Pernet RB. 1959. Les plantes médicinales malgaches. Mémoires de I'Institut Scientifique de Madagascar B(IX):219-299.

Rabearivony DA. 2010. Etude ethnobotanique des espèces médicinales à Ambalabe-Vatomandry et évaluation de leur structure écologiques. Mémoire de DEA, Faculté des Sciences. Antananarivo.

Rabefiraisana HJ. 2011. Evaluation du taux de mycorhisation des plantes médicinales et aromatiques de Madagascar. Mémoire de DEA option Physiologie Végétale. Faculté des Sciences. Université d'Antananarivo.

Rabemiafara MV. 2014. Ethnobotanique, écologie et statut de conservation de Aloe macroclada Baker dans la Région d'Analamanga. Mémoire DEA, Ecologie Végétale, Faculté des Sciences, Université d'Antananarivo.

Rabesa ZA. 1986. Pharmacopée de l'Alaotra, Ed. Fanantenana Antananarivo.

Rabesandratana RN. 1977. Résultats d'enquête et de localisations de plantes médicinales de la région de Tuléar. Annales de l'Université de Madagascar, Série Sciences de la Nature et des Mathématiques 13:131-150.

Rafidison V. 2013. Ethnobiologie et écologie des Ficus des terroirs Betsileo et du corridor Ranomafana- Andringitra. Thèse de Doctorat, Biologie et Ecologie Végétales, Faculté des Sciences, Université d'Antananarivo.

Rakotoarison-Ramiliarisoa B. 1993. L'agriculture traditionnelle et les utilités des plantes dans le NordEst Malgache. Thèse de Doctorat en biologie végétale tropicale, Université Paris 6.

Rakotoarivelo $\quad \mathrm{NH}, \quad$ Rakotoarivony $\mathrm{F}$, Ramarosandratana AV, Jeannoda V, Kuhlman AR, Randrianasolo A, Bussmann RW. 2015. Medicinal plants used to treat the most frequent diseases encountered in Ambalabe rural community, Eastern Madagascar. Journal of Ethnobiology and Ethnomedecine. doi :10.1186/s13002-015-0050-2.

Rakotobe EA, Rasolomanana CJC, Randrianasolo SS. 1993. Pharmacopées de l'Ambongo et de Boina, CIDST Antananarivo.

Rakotonandrasana SR, Rakotondrafara A, Rakotondrajaona R, Rasamison V, Ratsimbason M. 2017. Plantes médicinales des formations végétales de la Baie de Rigny Antsiranana à Madagascar, Bois et forêt des tropiques 331:55-65.

Rakotonandrasana SR. 2013. Les plantes médicinales de l'aire protégée de Zahamena (Madagascar) et ses environs: richesse floristique et endémicité, Scripta Botanica Belgica 50:356-362.

Rakotonandrasana SR, Oskolski AA,Tilney PM, Ekaterina L, Kotina EL, van Wyk B.-E. 2017. The rediscovery of Billburttia vaginoides, with notes on the morphology, anatomy, traditional uses and conservation status of the genus Billburttia (Apieae, Apiaceae). Phytotaxa 321(3):265-276. 
Rakotondrabe I. 2011. Caractérisation écologique, étude ethnobotanique statuts de conservation de quelques espèces du genre Uncarina (Pedaliaceae), endémique de Madagascar. Mémoire de DEA, Faculté des Sciences. Université d'Antananarivo.

Rakotondrafara A. 2010. Etudes écologiques, ethnobotaniques et évaluation des statuts UICN des huit espèces du genre Cedrelopsis endémique de Madagascar (RUTACEAE). Mémoire de DEA, Ecologie Végétale, Faculté des Sciences, Université d'Antananarivo.

Rakoto-Ratsimamanga A, Boiteau P, Mouton M. 1969. Eléments de pharmacopée Malagasy, tome I, Notice 1 à 39 , Société pour la promotion de la pharmacopée malagasy, IMRA. Tananarive.

Ramamonjihasina MM. 2013. Les mangroves de la station forèstière d'Antrema : Ecologie, menaces et pressions, utilisations locales. Mémoire de fin d'étude pour l'obtention du diplôme de licence professionnelle en conservation et valorisation de la biodiversité, Université de Fianarantsoa.

Ranarijaona HLT, Tsitomotra A, Ravoniarisoa JB, Andrianarisetra GS. 20013 Les plantes magiques traditionnelles les plus réputées des femmes de la ville de Mahajanga. Tela Botanica.

Randriamahefa M, Rakotozafy A. 1979. Tari-dalàna ahafantarana ny raokandro malagasy, Boky voalohany.

Randriamiharisoa MN, Kuhlman AR, Jeannoda V, Rabarison H, Rakotoarivelo N. Randrianarivony $\mathrm{T}$, Rakotoarivony F, Randrianasolo A, Bussmann RW. 2015. Medicinal plants sold in the markets of Antananarivo, Madagascar.Journal of Ethnobiology and Ethnomedecine. doi:10.1186/s13002-015-0046y.

Randrianarivony TN, Ramarosandratana AV, Andriamihajarivo $\mathrm{TH}$, Rakotoarivony $\mathrm{F}$, Jeannoda VH, Randrianasolo A, Bussmann RW. 2017. The most used medicinal plants by communities in Mahaboboka, Amboronabo, Mikoboka, Southwestern Madagascar. Journal of Ethnobiology and Ethnomedicine.

Randrianarivony T, Randrianasolo A, Andriamihajarivo $\mathrm{T}$, Ramarosandratana AV, Jeannoda VH, Rakotoarivony F, Bussmann RW. 2016. Useful plants and tradition for pregnancy, child delivery and for post-partum care used by people living around Analavelona forest in South west Madagascar. Indian Journal of Traditional Knowledge 15:68-78.

Randrianiaina M. 2012. Inventaire des plantes médicinales des forêts aux alentours des zones de conservation du projet Ambatovy en vue de leur valorisation. Mémoire de fin d'étude d'Ingénieur en
Sciences agronomiques Option Eaux et Forêts. ESSA Antananarivo.

Randrianjafy NEE. 2017. Etudes écologiques et modes d'utilisations des plantes médicinales de la nouvelle aire protégée de Maromizaha. Mémoire DEA, Ecologie Végétale, Faculté des Sciences, Université d'Antananarivo.

Rakotonandrasana SR, Randrianasolo SS 2006. Les plantes médicinales de l'AnkaranaFarafangana. Rapport Annuelle du CNARP.

Rakotonandrasana SR, Rakotondrafara A, Rakotondrajaona R, Rasamison V, Ratsimbason M. 2017. Plantes médicinales des formations végétales de la baie de Rigny- Antsiranana à Madagascar. Bois et Forêts des Tropiques 331(1):55-65.

Rasolohery A, Andriamiarisoa RL. 2006. Inventaire des Fougères de Zahamena, Madagascar. Missouri Botanical Garden. Saint Louis.

Ratefinjanahary J, Razafindraibe J, Randriamananjara L. 2000. Les plantes médicinales dans le corridor Fandriana Marolambo. Tableau de Bord Environnemental Région Amoron'i Mania. Ministére de l'environnement, des eaux et forêts, Office National pour l'environnement, Madagascar.

Ratsaralaza HL. 2010. Les plantes médicinales les plus utilisées de la nouvelle Aire protégée d'Agnalazaha (Etudes ethnobotaniques et écologiques en vue de l'élaboration d'une stratégie de conservation. Mémoire de DEA en Ecologie végétale appliquée. Faculté des Sciences, Antananarivo.

Ratsimiala-Ramonta I. 2010. Contribution de l'Ethnobotanique dans la médecine traditionnelle malgache, mémoire de HDR, Faculté des Sciences.

Ravaosolo J. 2009.Talismans utilisés en phytotérapie dans le Sud-Ouest malgache, le cas de Toliara. Etude Océan Indien, 42-43.

Raveloarimalala ML. 2011. Valeur d'usage, perception paysanne, ménace et pression sur les Bambous du Sud-Ouest de Madagascar: cas d'Andranomaitso (Sakaraha) et Antanisoa (Betioky Sud). Mémoire DEA, Ecologie Végétale, Faculté des Sciences, Université d'Antananarivo.

Ravelontsoa F. 2010. Valorisation de la biodiversité végétale de la Région Sofia: étude ethnobotanique, phytochimique et pharmacologique de Fuirena glomerata Lam (Cyperaceae). Mémoire de Master II, Faculté des sciences, Université de Mahajanga.

Razafiarisoa VH. 2016. Etat de lieux environementaux des environs du massif de Vohimbohitra en vue d'un plan de conservation, Mémoire pour l'obtention du diplôme de master, Faculté des Sciences, Université d'Antananarivo. 
Razafimahatratra TF. 2018. Formations végétales à Uapacca bojeri de la commune rurale de Ramainandro, District de Faratsiho. Mémoire de licence professionnelle en Gestion de l'Environnement, Mention biologie, Institut d'Enseignement Supérieur AntsirabeVakinankaratra.

Razafindraibe M, Kuhlman AR, Rabarison $H$, Rakotoarimanana $\mathrm{V}$, Rajeriarison $\mathrm{C}$, Rakotoarivelo $\mathrm{N}$, Randrianarivony T, Rakotoarivony F, Ludovic R, Randrianasolo A, Bussmann RW. 2013. Medicinal plants used by women from Agnalazaha littoral forest (Southeastern Madagascar). Journal of Ethnobiology and Ethnomedicine 9:13

Razafindrazaka RM. 2012. Les plantes sauvages les plus utilisées dans la Région Analamanga : Inventaire ethnobotanique dans les communes rurales d'Ankadinandriana, d'Ambohitrandriamanitra et de Miadanandriana. Mémoire CAPEN, Ecole Normale Supérieure, Université d'Antananarivo.

Razaindrafy ML. 2008. Evaluation de l'importance de plantes médicinales et les autres produits secondaires. Cas du Fokontany Amindrabe, Commune Rurale d'Androy District de Lalangina Région de Haute Matsiatra. Mémoire de fin d'étude. Univesité de Fianarantsoa.

Safidiniaina A. 2018. Plantes médicinales des formations herbeuses de la commune rurale de Ramainandro, district de Faratsiho. Mémoire de licence professionnelle en Gestion de l'Environnement, Mention biologie, Institut d'Enseignement Supérieur AntsirabeVakinankaratra.

Schmitt J P. 1971. Contribution à l'inventaire des plantes médicinales de Madagascar, ORSTOM, Section des plantes médicinales, $\mathrm{V}$, Tananarive.

Tida MAM. 1996. Contribution à l'inventaire forestière et à l'étude de la flore médicinale des relictes forestières de Tsinjoarivo. Mémoires de DEA, Faculté des Sciences Université d'Antananarivo.

Vonimboahirana CG. 2008. Contribution à l'étude des plantes à usage multiple cas de village d'Andohanisoa, Commune rurale de Miarinarivo, District d'Ambalavao, Région Haute-Matsiatra. Mémoire DTS en Environnement, Institut des Sciences et Techniques de l'Environnement, Université de Fianarantsoa.

Wieederkehr S, Thiébaut L, Callmander MW, Wohlhauser S, Kupfer P. 2005. Pharmacopée et médecine traditionnelle dans la penninsule de Masoala. Bulletion de la Société Neuchâteloise des Sciences Naturelles. Tome 128.
Yvon C, Chabouis L, Chabouis F. 1970. Végétaux et groupements végétaux de Madagascar et des Mascareignes, Tome 1. Bureau pour le Développement de la Production Agricole. Antananarivo.

Yvon C, Chabouis L, Chabouis F. 1970. Végétaux et groupements végétaux de Madagascar et des Mascareignes, Tome 2. Bureau pour le Développement de la Production Agricole. Antananarivo.

Yvon C, Chabouis L, Chabouis F. 1970. Végétaux et groupements végétaux de Madagascar et des Mascareignes, Tome 3. Bureau pour le Développement de la Production Agricole. Antananarivo.

Yvon C, Chabouis L, Chabouis F. 1969. Végétaux et groupements végétaux de Madagascar et des Mascareignes, Tome 4. Bureau pour le Développement de la Production Agricole. Antananarivo.

Zitt F. 2000. Etude préliminaire à l'élaboration du programme de valorisation du Katrafay (Cedrelopsis grevei) et des plantes médicinales de la région de Fort-Dauphin - CNRE, Antananarivo. 